\title{
Propensity-score-matched Evaluation of Psychological Stress and Hair Cortisol among People Living with HIV in China
}

\section{Xu Chen}

People's Hospital of Guigang City

\section{Cheng-Bo Zeng}

University of South Carolina

\section{Shuai-Feng Liu}

Guangxi Center of Disease Control and Prevention

\section{Xiao-Ming Li}

University of South Carolina

\section{Shan Qiao}

University of South Carolina

Ri-Ying Lv ( $\nabla$ lvriying@163.com )

People's Hospital of Guigang City

\section{Zhi-Yong Shen}

Guangxi Center of Disease Control and Prevention

\section{Research Article}

Keywords: psychological stress, hair cortisol level, HIV (PLWH)

Posted Date: December 22nd, 2020

DOl: https://doi.org/10.21203/rs.3.rs-124276/v1

License: (9) This work is licensed under a Creative Commons Attribution 4.0 International License.

Read Full License 


\title{
Propensity-score-matched evaluation of psychological stress and hair
}

\section{cortisol among people living with HIV in China}

\author{
Xu Chen ${ }^{1}$, Cheng-Bo Zeng ${ }^{2}$, Shuai-Feng Liu ${ }^{3}$, Xiao-Ming $\mathrm{Li}^{2}$, Shan Qiao ${ }^{2}$, Ri-Ying $\mathrm{Lv}^{{ }^{*}}$, Zhi-Yong Shen ${ }^{3^{*}}$ \\ ( ${ }^{1}$ Department of Infectious Disease, People's Hospital of Guigang City, Guigang, Guangxi, China; \\ ${ }^{2}$ Department of Health Promotion Education and Behavior, School of Public Health, University of South \\ Carolina, Columbia, South Carolina, United States of America; ${ }^{3}$ Guangxi Center of Disease Control and \\ Prevention, Nanning, Guangxi, China. E-mail: lvriying@163.com; shenzhiyong99999@sina.com)
}

\begin{abstract}
To compare the psychological stress level and hair cortisol level of people living with HIV (PLWH) with those without HIV. A total of 220 subjects were initially enrolled in the study, including 20 subjects living without HIV and 200 PLWH. Psychological stress level, including quality of life, anxiety, perceived stress and mental resilience, was self-reported in both groups with related scales. The cortisol in hair were extracted and assessed by LC-APCI-MS/MS method. Propensity score matching analysis was performed to balance the baseline covariates of the two groups, whereas the difference in psychological stress level and hair cortisol level between the two groups were compared. Furthermore, the associations between psychological stress level and cortisol level were examined. Two comparison groups were matched by 1:3 propensity score matching, which yield 20 subjects living without HIV and 60 PLWH. Ultimately, in regarding to the psychological stress, the levels of the anxiety, perceived stress and mental resilience were higher among PLWH than those living without HIV, but the people without HIV showed higher quality of life. The hair cortisol level in PLWH was higher than those living without HIV. However, there were no significant associations between psychological stress level and cortisol level. The PLWH showed higher level of psychological stress and cortisol than those without HIV. No relationship was seen between psychological stress level and cortisol level in PLWH.
\end{abstract}

\section{Introduction}

Human immunodeficiency virus (HIV), which causes Acquired Immunodeficiency Syndrome (AIDS), affects up to $0.8 \%$ of the adult worldwide. Over the past decades, international donor institutions and governments have allocated substantial resources to control the HIV/AIDS epidemic. Despite the significant progress in this process, the epidemic still represents a significant public health threat ${ }^{1}$, with an estimated 37.9 million of PLWH by the end of 2018 worldwide ${ }^{2}$.The number of HIV-infected patients is still rising in China and other parts of the world. With the antiretroviral therapy (ART), there was a significant decline in the incidence of opportunistic infection- and neoplasm- related diseases. Nevertheless, psychological problems, including anxiety, perceived stress and stigma, as well as HIV-related endocrinopathies seem to become increasingly important among $\mathrm{PLWH}^{3}$. Previous studies have shown that nearly $50 \%$ of the PLWH suffer from various psychological problems at all stages of HIV infectio ${ }^{4}$. The high levels of psychological distress would generate physiological reactions (e.g. cortisol, Dehydroepiandrosterone, catecholamine) and harm the physical health of PLWH.

Cortisol, as one end product of the hypothalamic-pituitary-adrenal (HPA) axis, has been widely used as one of the main indicators of cumulative physiological responses associated with psychological distress ${ }^{5,6}$. These responses are generated when people suffer from emergent external stress and help them adapt to the special environmental changes. Cortisol could be 
obtained from the blood, saliva, and urine samples. Because these common sampling matrices provide measures of cortisol concentration at single points in time, they are likely affected by the time of the day samples are collected, individual differences in circadian rhythms, and transient exposure to acute daily stressors. Compared with traditional measurement, hair cortisol is relatively stable and could capture the long-term physiological responses related to stress. What's more, hair samples can be stored for long periods and delivered without biohazardous precautions or cold chain requirements ${ }^{7}$. Therefore, hair cortisol measurement is likely to overcome the shortcoming mentioned above. It is not surprising that hair cortisol is a potentially promising biomarker of psychological distress.

Therefore, we conducted this study to investigate the psychological stress level and cortisol level among PLWH from China. In contrast to previous studies using single time point (saliva, serum) or short-term ( $24 \mathrm{~h}$ urine) cortisol measurements, we used a relatively novel but well-validated method to determine long-term systemic cortisol levels in scalp hair ${ }^{8}$. Importantly, psychological stress and hair cortisol level are likely to be affected by various confounders, such as age, gender, body mass index (BMI), and ethnicity, which can bias the results. We therefore performed propensity score matching (PSM) analysis to balance baseline covariates between the groups of PLWH and people living without HIV to examine the relationship between HIV infection and psychological distress, as well as level of cortisol. The associations between psychological distress and cortisol were examined as well.

\section{Results}

\section{Baseline characteristics}

A total of 220 participants were enrolled in the study, including 20 subjects living without HIV and 200 PLWH. Among them, 137(62.27\%) of the participants were males while 83(37.73\%) were females. The median age was 41.73(IRQ: 33.94-48.68) years old and the median BMI was 21.30(IRQ: 19.77-23.82). Before matching, the baseline characteristics between the two groups that differed significantly in terms of ethnicity, monthly household income and alcohol use $(\mathrm{P}<$ 0.05 ). We used the propensity score matching method to balance the differences between the study and control groups. The 1:3 PSM yielded matched pairs of 20 participants in the control group and 60 PLWH in the study group. No statistical differences were observed between the two groups with $\mathrm{p}>0.05$ for all baseline variables after matching (Table 1). In order to further study this imbalance, histograms were used to display the propensity score distribution before and after PSM. Figure 1a presents a histogram showing an unbalanced distribution of propensity scores for the overall participants, whereas Figure $1 \mathrm{~b}$ presents the tendency for equilibrium of the score distribution in the histogram for the matched participants.

Table 1.Baseline characteristics before and after propensity matching in control and study groups

\begin{tabular}{lcccccc}
\hline Characteristics & & $\begin{array}{c}\text { Before } \\
\text { propensity } \\
\text { matching }\end{array}$ & & & & \multicolumn{3}{c}{$\begin{array}{c}\text { After } \\
\text { propensity } \\
\text { matching }\end{array}$} \\
\hline & $\begin{array}{c}\text { Control group } \\
\mathrm{N}=20\end{array}$ & $\begin{array}{c}\text { Study group } \\
\mathrm{N}=200\end{array}$ & $\begin{array}{c}\text { P } \\
\text { value }\end{array}$ & $\begin{array}{c}\text { Control group } \\
\mathrm{N}=20\end{array}$ & $\begin{array}{c}\text { Study group } \\
\mathrm{N}=60\end{array}$ & $\begin{array}{c}\mathrm{P} \\
\text { value }\end{array}$ \\
\hline $\begin{array}{l}\text { Age (median, } \\
\text { IQR) }\end{array}$ & $39(32.8-44.3)$ & $42(34.1-49.1)$ & 0.088 & $39(32.8-44.3)$ & $37(31-47.3)$ & 0.534 \\
Gender & & & $\mathbf{0 . 4 8 2}$ & & & $\mathbf{1 . 0 0 0}$ \\
\hline
\end{tabular}




\begin{tabular}{|c|c|c|c|c|c|c|}
\hline Male & $11(55 \%)$ & $126(63 \%)$ & & $11(55 \%)$ & $33(55 \%)$ & \\
\hline Female & $9(45 \%)$ & $74(37 \%)$ & & $9(45 \%)$ & $27(45 \%)$ & \\
\hline $\begin{array}{l}\text { BMI(median, } \\
\text { IQR) }\end{array}$ & $21.3(19.7-22.6)$ & $21.3(20.0-24.0)$ & 0.857 & $\begin{array}{c}21.3 \\
(19.7-22.6)\end{array}$ & $\begin{array}{c}21.2 \\
(19.8-22.6)\end{array}$ & 0.874 \\
\hline Ethnicity & & & 0.030 & & & 1.000 \\
\hline Han & $19(95 \%)$ & $146(73 \%)$ & & $19(95 \%)$ & $55(91.7 \%)$ & \\
\hline Non-Han & $1(5 \%)$ & $54(27 \%)$ & & $1(5 \%)$ & $5(8.3 \%)$ & \\
\hline Marital status & & & 0.427 & & & 1.000 \\
\hline Married & $16(80 \%)$ & $173(86.5 \%)$ & & $16(80 \%)$ & $50(83.3 \%)$ & \\
\hline Never married & $4(20 \%)$ & $27(13.5 \%)$ & & $4(20 \%)$ & $10(16.7 \%)$ & \\
\hline Education level & & & 0.614 & & & 0.700 \\
\hline $\begin{array}{l}\text { Illiterate or } \\
\text { primary school }\end{array}$ & $5(25 \%)$ & $70(35 \%)$ & & $5(25 \%)$ & $17(28.3 \%)$ & \\
\hline $\begin{array}{l}\text { Junior high } \\
\text { school }\end{array}$ & $10(50 \%)$ & $85(42.5 \%)$ & & $9(45 \%)$ & $28(46.7 \%)$ & \\
\hline $\begin{array}{l}\text { Senior high } \\
\text { school }\end{array}$ & $3(15 \%)$ & $34(17 \%)$ & & $4(20 \%)$ & $13(21.7 \%)$ & \\
\hline College or above & $2(10 \%)$ & $11(5.5 \%)$ & & $2(10 \%)$ & $2(3.3 \%)$ & \\
\hline $\begin{array}{l}\text { Employment } \\
\text { status }\end{array}$ & & & 0.082 & & & NA \\
\hline Unemployed & 0 & $18(9 \%)$ & & 0 & 0 & \\
\hline $\begin{array}{l}\text { Part time } \\
\text { employed }\end{array}$ & 0 & $24(12 \%)$ & & 0 & 0 & \\
\hline $\begin{array}{l}\text { Full time } \\
\text { employed }\end{array}$ & $20(100 \%)$ & $158(79 \%)$ & & $20(100 \%)$ & $60(100 \%)$ & \\
\hline Monthly & & & & & & \\
\hline $\begin{array}{l}\text { household } \\
\text { income(Yuan) }\end{array}$ & & & 0.001 & & & 0.270 \\
\hline$<2000$ & 0 & $32(16 \%)$ & & 0 & 0 & \\
\hline 2000-2999 & $4(20 \%)$ & $95(47.5 \%)$ & & $4(20 \%)$ & $22(36.7 \%)$ & \\
\hline$\geq 3000$ & $16(80 \%)$ & $73(36.5 \%)$ & & $16(80 \%)$ & $38(63.3 \%)$ & \\
\hline Tobacco use & & & 0.064 & & & 0.636 \\
\hline Yes & $3(15 \%)$ & $71(35.5 \%)$ & & $3(15 \%)$ & $14(23.3 \%)$ & \\
\hline No & $17(85 \%)$ & $129(64.5 \%)$ & & $17(85 \%)$ & $46(76.7 \%)$ & \\
\hline Alcohol use & & & 0.012 & & & 0.819 \\
\hline Yes & $1(5 \%)$ & $64(32 \%)$ & & $1(5 \%)$ & $6(10 \%)$ & \\
\hline No & $19(95 \%)$ & $136(68 \%)$ & & $19(95 \%)$ & $54(90 \%)$ & \\
\hline $\begin{array}{l}\text { Frequency of } \\
\text { hair washing }\end{array}$ & & & 0.746 & & & 1.000 \\
\hline $\begin{array}{l}\text { Once every } 1 \text { to } 3 \\
\text { days }\end{array}$ & $18(90 \%)$ & $175(87.5 \%)$ & & $18(90 \%)$ & $52(86.7 \%)$ & \\
\hline $\begin{array}{l}\text { Once every } 4 \text { to } 7 \\
\text { days }\end{array}$ & $2(10 \%)$ & $25(12.5 \%)$ & & $2(10 \%)$ & $8(13.3 \%)$ & \\
\hline Using Hair & & & 0.895 & & & 0.896 \\
\hline
\end{tabular}


dryer, curling

iron or hair

straightener

Yes

$8(40 \%)$

$77(38.5 \%)$

$8(40 \%)$

$27(45 \%)$

No

$12(60 \%)$

$123(61.5 \%)$

$12(60 \%)$

$33(55 \%)$

Using hair styling products

0.261

NA

\begin{tabular}{lcccc} 
Yes & 0 & $12(6 \%)$ & 0 & 0 \\
No & $20(100 \%)$ & $188(94 \%)$ & $20(100 \%)$ & $60(100 \%)$ \\
\hline
\end{tabular}

Data are presented as $\mathrm{n}(\%)$ or median $\left(25^{\text {th }}-75^{\text {th }}\right.$ interquartile range $)$.

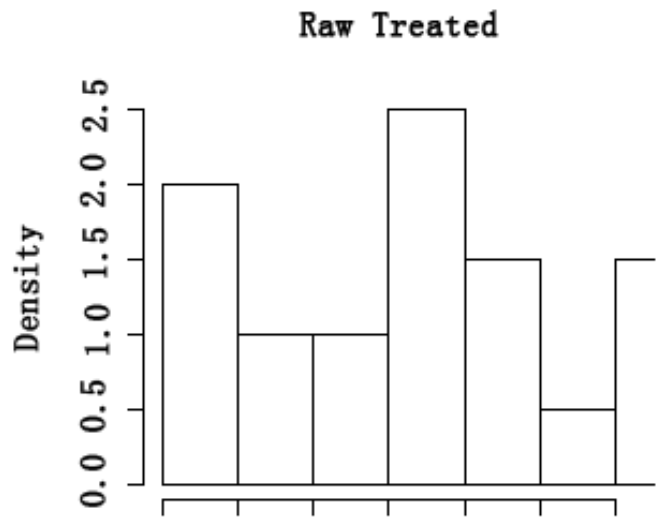

$\begin{array}{lllllll}0.0 & 0.1 & 0.2 & 0.3 & 0.4 & 0.5 & 0.6\end{array}$

Propensity Score

\section{Raw Control}

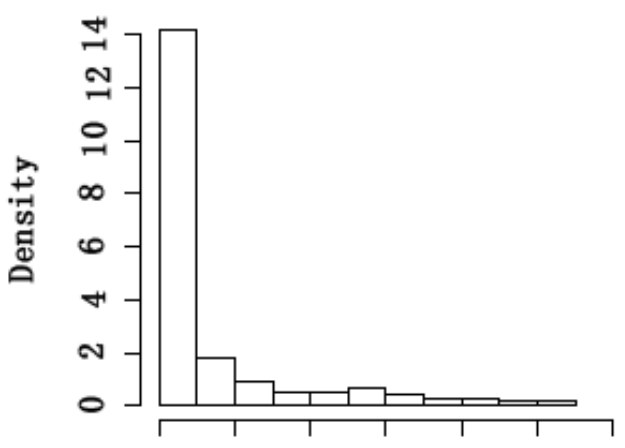

$\begin{array}{lllllll}0.0 & 0.1 & 0.2 & 0.3 & 0.4 & 0.5 & 0.6\end{array}$

Propensity Score
Matched Treated

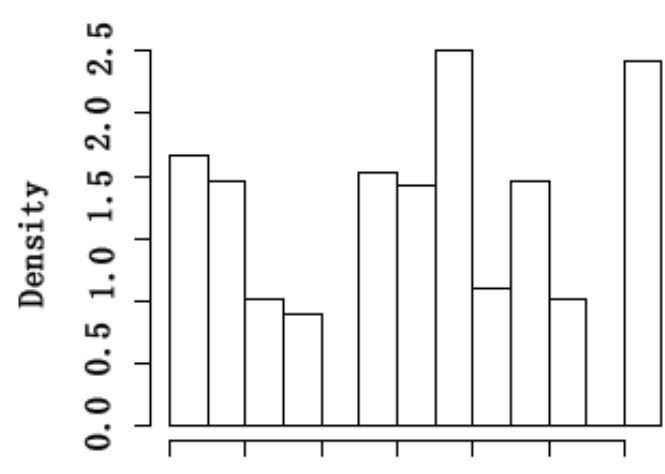

$\begin{array}{lllllll}0.0 & 0.1 & 0.2 & 0.3 & 0.4 & 0.5 & 0.6\end{array}$

Propensity Score

Matched Control

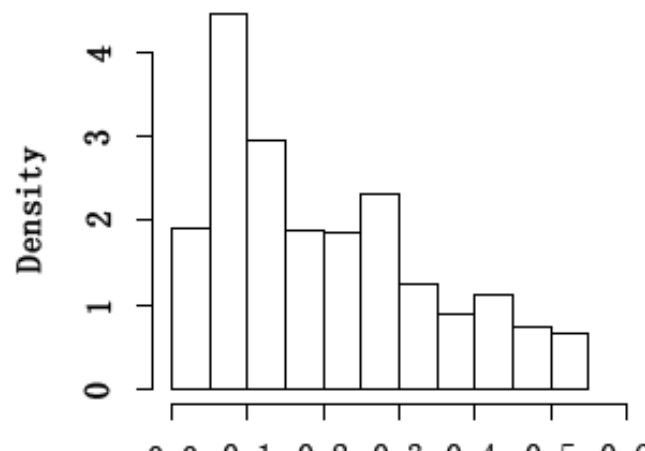

$\begin{array}{llllllll}0.0 & 0.1 & 0.2 & 0.3 & 0.4 & 0.5 & 0.6\end{array}$

Propensity Score

Figure 1 Histograms of the unmatched and matched participants.

Note: The overall balance of the thirteen variables in the model indicates that the matching process was successful.

\section{Comparison of psychological stress and hair cortisol between control and study groups}

In this study, we compared the level of psychological stress and hair cortisol between the study 
and control groups (Table 2). The medians of psychological stress are presented in Figure 2a-2d. Compared with control group, the study group reported significantly lower level of quality of life (109 vs. 116, p<0. 001). In addition, the study group reported significantly higher levels of anxiety ( 34 vs. $26, p<0.001$ ), perceived stress ( 38.5 vs. $33, p=0.001)$ and psychological resilience ( 31 vs. 26, $\mathrm{p}=0.004$ ) than control group. In PLWH, their median of hair cortisol was 34.66 (IQR: 21.74-61.93), whereas for the participants in the control group, their median of hair cortisol was 21.61(IQR: 14.83-26.75), and the difference in hair cortisol was statistically significant between the two groups $(\mathrm{P}=0.002)$ (Figure 2e).

Table 2 Comparison of psychological stress and hair cortisol between control and study groups

\begin{tabular}{lllll}
\hline & Control group & Study group & Z value & P value \\
\hline Psychological measure & & & & \\
Quality of life & 116(115-118.25) & 109(104-114.25) & -3.545 & 0.000 \\
Anxiety & 26(25.75-29) & $34(31-36)$ & -4.722 & 0.000 \\
Perceived stress & $33(30.75-37)$ & $38.5(34.75-42)$ & -3.323 & 0.001 \\
Psychological resilience & 26(23-28.5) & $31(27-34)$ & -2.844 & 0.004 \\
Endocrine measure & & & & \\
Cortisol & $21.61(14.83-26.75)$ & $34.66(21.74-61.93)$ & -3.089 & 0.002 \\
\hline
\end{tabular}

a

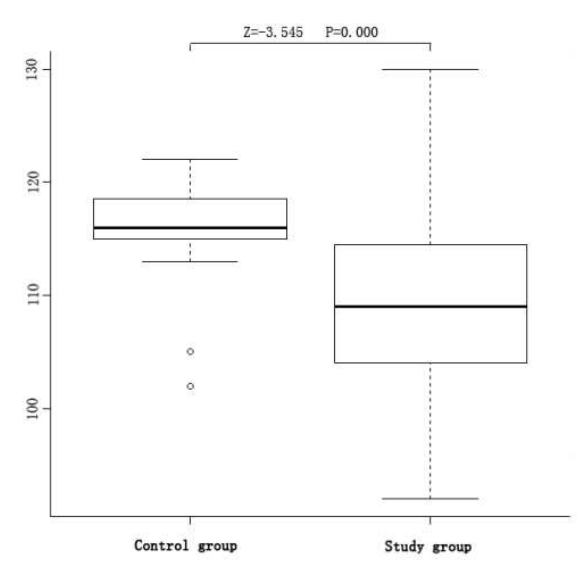

b

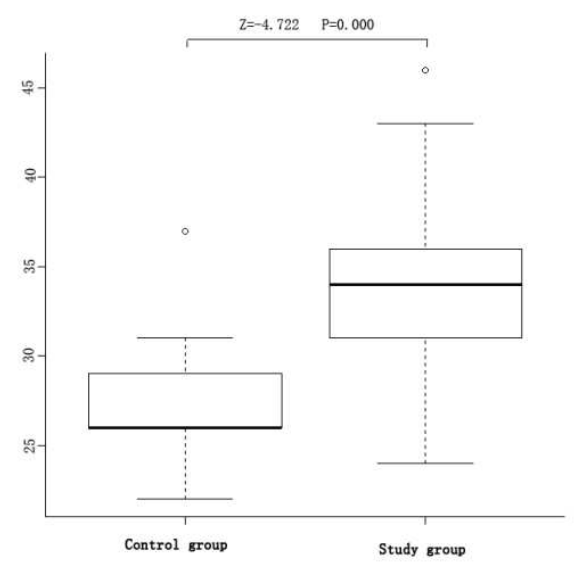



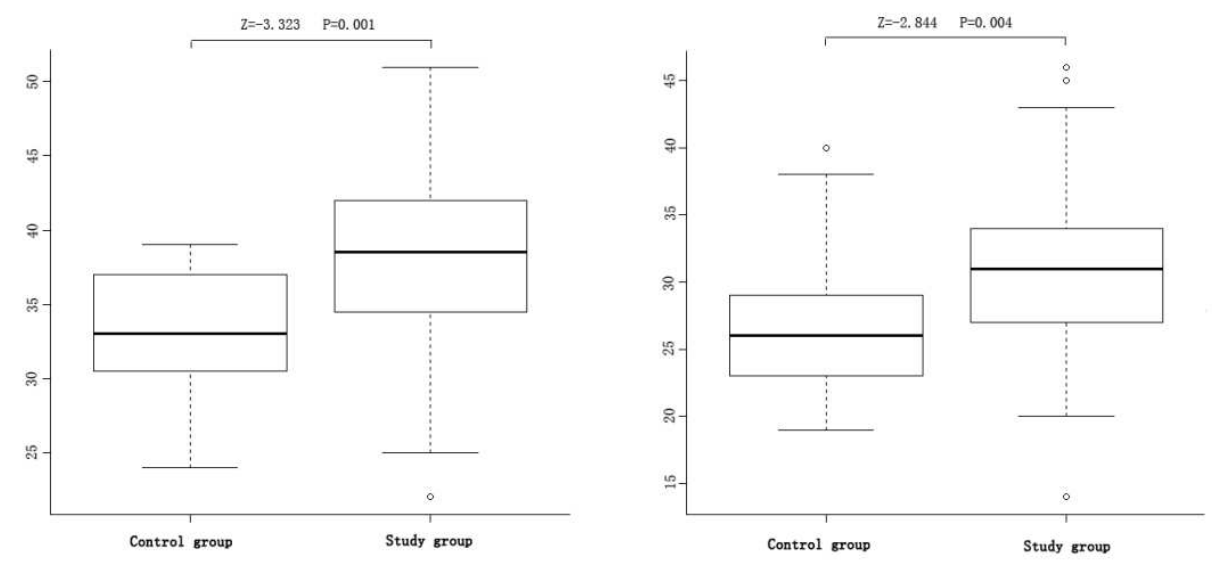

e

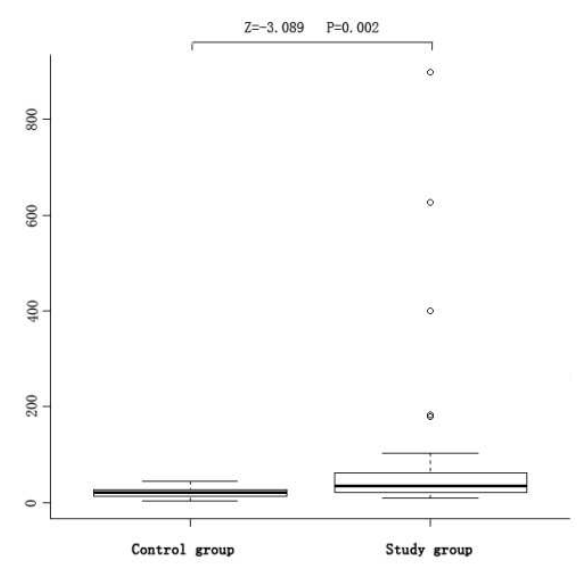

Figure 2.Comparison of psychological stress level and cortisol level between control and study groups after propensity matching. (a-d) The level of quality life, anxiety, perceived stress, and psychological resilience among PLWH and without HIV, respectively. (e) The level of cortisol among PLWH and without HIV.

\section{The associations between psychological stress and cortisol}

According to Spearman correlation analyses, hair cortisol was negatively related to quality of life $(\mathrm{r}=-0.063, \mathrm{p}=0.378)$ and perceived stress $(\mathrm{r}=-0.043, \mathrm{p}=0.542)$. However, it was positively related to anxiety $(\mathrm{r}=0.061, \mathrm{p}=0.389)$ and mental resilience $(\mathrm{r}=0.082, \mathrm{p}=0.249)$. But none of these associations were statistically significant.

Medians for each psychological stress by hair cortisol level are illustrated in Table 3. Hair cortisol level was negatively associated to quality of life $(\mathrm{z}=-0.005, \mathrm{p}=0.996)$, anxiety $(\mathrm{z}=-0.724, \mathrm{p}=0.469)$, perceived stress $(\mathrm{z}=-5.04, \mathrm{p}=0.614)$ and psychological resilience $(\mathrm{z}=-1.139, \mathrm{p}=0.255)$ although no significant association were detected.

Table 3 Psychological stress by level of hair cortisol

\begin{tabular}{lllll}
\hline Cortisol & Quality of life & Anxiety & Perceived stress & $\begin{array}{l}\text { Psychological } \\
\text { resilience }\end{array}$ \\
\hline High $(\leqslant 29.56)$ & $109.5(105-115.25)$ & $34(30-36)$ & $38.5(35-42)$ & $31(28-34)$ \\
Low $(>29.56)$ & $110(105-115)$ & $33(30-36)$ & $38(32-41)$ & $29.5(27-34.75)$ \\
\hline
\end{tabular}




\begin{tabular}{lllll}
\hline$Z$ value & -0.005 & -0.724 & -5.04 & -1.139 \\
$P$ value & 0.996 & 0.469 & 0.614 & 0.255 \\
\hline
\end{tabular}

\section{Discussion}

This study compared the psychological distress and hair cortisol between PLWH and people living without HIV from Guangxi, China, and showed that PLWH displayed higher levels of psychological stress and hair cortisol. Moreover, we found that the PLWH presented lower quality of life but higher psychological resilience. These results are consistent with that of the existing literature ${ }^{9,10}$. As we know, psychological distress and hair cortisol are likely to be affected by various confounders. The propensity score matching analysis was used in our study to minimize the confounding factors as far as possible. A previous study has suggested that matching according to the propensity score eliminates a greater proportion of baseline difference between any two groups compared to stratification or covariate adjustment ${ }^{11}$ The 1-to-3 propensity score matching provides a robust evaluation of psychological stress and hair cortisol among PLWH.

The results in this study revealed that PLWH showed higher level of psychological stress compared with the general population, which is the same as seen in previous study ${ }^{12}$. A study from the United States showed that about 56\% - 78\% of AIDS patients suffer from mental disorders. Depression, anxiety, fear, loss of confidence in life, suicidal behaviors are common psychological problems among them ${ }^{13}$. A large number of research indicated that the PLWH presented significant lower level of psychological health than those without HIV ${ }^{14,15}$. Most PLWH must cope with the complex and multiple psychological problems of a life-threatening illness and its treatment, which simultaneously facing financial hardship, life-style associated social stigmas, multiple bereavements, and other personal losses ${ }^{16}$. With the high prevalence of these stressors, it is not surprising that PLWH are at increased risk for affective disorders and sustained high levels of psychological stress.

Our study also found that the PLWH displayed higher level of hair cortisol than those without HIV. The reason is, on the one hand, that HIV can activate monocytes-macrophages to produce cytokines including interleukin-1, tumor necrosis factor, interferon and then directly or indirectly influence the HPA to stimulate the secretion of cortisol $^{10,17}$. On the other hand, cortisol is an important indicator of chronic stress. As mentioned above, PLWH have high levels of psychological distress, which promote the secretion of adrenocorticotrophic hormone resulting in the increased cortisol level ${ }^{18}$. In contrast, some studies indicated that $8 \%-14 \%$ PLWH were observed lower level of cortisol than normal level ${ }^{17,19}$. It was likely to relate to activation of polyclonal $\beta$ cell, development of anti-adrenal cell antibody and adrenal dysfunction ${ }^{20}$. Other factors including diet, sports and drugs, which were difficult to be excluded, may also affect the synthesis of cortisol as well.

Cortisol is commonly known as the stress hormone because it is released in higher doses under stressful condition. For instance, some studies indicated that hair cortisol increased with higher level of stressful life events, depression, and posttraumatic stress disorder ${ }^{21-23}$. In the same way, one systematic literature review on hair cortisol and stress exposure indicated that effect sizes for the connections between hair cortisol and chronic stress were mostly medium and large ${ }^{24}$. However, although PLWH had higher level of psychological stress and cortisol than the control group, no significant association between psychological stress and hair cortisol was found in the study, which was consistent with previous studies ${ }^{25,26}$. Some studies even reported that anxiety 
was negatively associated with hair cortisol ${ }^{27}$.

Several limitations should be recognized in this study. First, the small sample size in this study might limit the statistical power of the analysis. Second, the cortisol and psychological situation were affected by various confounders. Although we used PSM to balance some of the sociodemographic characteristics between study and control groups, we could not completely exclude the influence of all confounders. Third, the study may have suffered from nonrespondents bias as there were some participants who refused to participate in the study ${ }^{28}$. Nevertheless, the study enabled us to understand the psychological distress and hair cortisol between the PLWH and the people without HIV in China. These findings may have implications for mental intervention and HIV-related endocrinopathies targeted at HIV-infected population. Special attention focused on psychological distress and cortisol should be given to PLWH. Though no relationship between psychological stress and cortisol was found in this study, hair cortisol analysis still hold great promise to accurately assess long-term levels of psychological distress, which could greatly contribute to early diagnosis and intervention of psychological health diseases and the improvement of mental wellbeing among PLWH. Further research is needed to better understand psychological illness and endocrine issues among the PLWH. Further studies investigating the relationship between the psychological stress and the cortisol are warranted as well.

In summary, our study confirms that the PLWH from China suffer from both higher levels of psychological stress and cortisol. Moreover, we find that the PLWH presented lower quality of life but higher psychological resilience. Further, no significant association was found between psychological stress and hair cortisol among PLWH in this study. The psychological distress and HIV-related endocrine system disease among PLWH are deserved more attention.

\section{Materials and methods}

The study protocol was approved by the Institutional Review Board of Guangxi. Written informed consent was obtained from participants. All of the procedures were performed in accordance with the Declaration of Helsinki and relevant policies in China.

\section{Subjects}

All participants of this study were recruited from Department of Infectious Disease in People's Hospital of Guigang City. Participants who were at least 18 but younger than 60 years of age were eligible for recruitment. The exclusion criteria were individuals who: 1) had confirmed symptoms of opportunistic infections; 2) endocrine diseases (e.g. macroamylasemia, adrenal dysfunction, hyperamylasemia, autoimmune thyroid diseases, etc.) ;3) currently take hormonal drugs (e.g. prednisolone) which might influen the cortisol level; 4) had a history of drug use; and 5) have other known diseases or comorbidities that could affect the cortisol level. Finally, we recruited 200 PLWH as the study group and 20 people who were HIV-negative but their spouses were HIV-positive as the control group.

\section{Data collection procedure}

Hair samples were collected from each participant by research staff from Department of Infectious Disease between November 2017 and April 2018. Guided by a standardized protocol as described previously $^{29}$, two $1-\mathrm{cm}$ hair samples (20-30 strands of hair) were cut as close to the scalp as possible from the vertex posterior region. The hair strands were cut with iron scissors which had been sanitized. The hair thatch, which was divided into two parts, was completely enclosed by a 
piece of foil with a small label indicating the study ID.

After the hair sample collection, all the participants must complete the survey to collect their sociodemographic characteristics and psychosocial outcomes (e.g. quality of life, anxiety, perceived stress and mental resilience). The information about hair care (e.g. frequency of hair washing, using hair dryer/curling iron or hair straightener or not, using hair styling products or not) were collected as well. Each interview lasted for 30 minutes and was performed through a one-on-one format. Interviewers were internal healthcare nurses who had received intensive training on research ethics and data collection with PLWH before the study.

\section{Cortisol assay}

Hair samples were washed twice with $1 \mathrm{~mL}$ methanol for 2 minutes and then dried at a room with temperature of $25^{\circ} \mathrm{C}$ for at least 12 hours. A sample of $20 \mathrm{mg}$ hair was then incubated in $1 \mathrm{~mL}$ methanol with $1 \mathrm{ng}$ cortisol-d4 as internal standard (IS) for 24 hours at $25^{\circ} \mathrm{C}$. After being centrifuged at $12,000 \mathrm{rpm}$ for 5 minutes, $800 \mu \mathrm{L}$ of the clear supernatant was transferred into a new $2 \mathrm{~mL}$ tube and evaporated under nitrogen at $50^{\circ} \mathrm{C}$. The dry residue was re-suspended using $50 \mu \mathrm{L}$ deionized water and $950 \mu \mathrm{L}$ methanol for solid-phase extraction. The final eluate was evaporated to dryness and re-suspended in $50 \mu \mathrm{L}$ mobile phase for the next analysis ${ }^{30}$.

The hair cortisol was tested using liquid chromatography tandem mass spectrometry (3200 QTRAP, ABI, USA). Cortisol was ionized with an atmospheric pressure chemical ionization source (APCI), and identified in positive ion mode using the multiple reaction monitoring mode and quantified with internal standard method. Nitrogen (99.999\%) was selected as the nebulizing gas. The optimum ionization parameters guided by the protocol were reported in previous study ${ }^{31}$. The current LC-APCI-MS/MS method showed the limits of detection (LOD) and quantification (LOQ) between 0.25 and $0.50 \mathrm{pg} / \mathrm{mg}$ for hair cortisol where LOD and LOQ were defined as the concentrations with a signal-to-noise ratio $(\mathrm{S} / \mathrm{N})$ between 3 and 10 . Linearity was achieved at $0.5-250 \mathrm{pg} / \mathrm{mg}(\mathrm{r} 2=0.9983)$ of hair cortisol. Intra-day and inter-day precisions and recovery $(\mathrm{n}=5)$ were evaluated at $0.5,5$ and $200 \mathrm{pg} / \mathrm{mg}$. Intra- day and inter-day coefficients of variation were less than $10 \%$ at the three concentrations and recovery was more than $98 \%$.

\section{Measure}

\section{Socio-demographic characteristics}

Participants provided information about their socio-demographic characteristics including age, gender, ethnicity, marital status, education level, employment status, monthly household income, tobacco use, alcohol use, and hair care (e.g. using hair_dryer/curling iron or hair straighteneror not, using hair styling products or not). Participant also reported their estimated weight and height. We calculated Body Mass Index (BMI) based on weight and height for each participant using an established formula BMI=weight $(\mathrm{kg}) /[\text { height }(\mathrm{m})]^{2}$.

\section{Psychological measures}

Quality of life

We employed Simplified Chinese Version of MOS-HIV Scale to evaluate the quality of live among all the participants. The MOS-HIV is a 35-item scale capturing 11 dimensions of health-related quality of life including physical functioning, role functioning, pain, social functioning, emotional well-being, energy/fatigue, cognitive functioning, general health, health distress, overall quality of life, and health transition. The summed score was calculated the quality of life, with higher score representing better functioning and well-being (Cronbach's $\alpha=0.938$ ).

Anxiety 
We employed the Zung Self-rating Anxiety Scale (SAS) to assess the levels of anxiety among participants. The SAS is a 20 -item scale that assesses symptoms of anxiety with a 4-point response option. The sample items are "how often do you feel scared for no reason" and "how often do you feel all right". SAS has been widely used in various Chinese populations and convincing internal reliability in the current study sample (Cronbach's $\alpha=0.93)^{32}$.

Perceived stress

Participants' subjective experience of chronic stress was measured using the 14-item Perceived Stress Scale (PSS) ${ }^{33}$, which has been validated in a wide variety of populations ${ }^{34}$. The PSS is a global measure of subjective stress that assesses the extent to which respondents perceive their lives as being unpredictable, uncontrollable, or overwhelming in the last month. Each item is rated on a 5-point scale ranging from "almost never" to "almost always" with higher scores indicating higher levels of perceived stress (Conbach's $\alpha=0.78$ ).

| Psychological resilience

The 10-item Connor-Davidson Resilience Scale (CD-RISC) was employed to evaluate the psychological resilience in this study. It assesses an individual's ability to adapt to the adversities in the past month. The response option is a 5-point Likert scale ranging from 0 (not true at all) to 4 (true nearly all the time). The summed score was calculated to reflect the resilience capacity, which higher score indicating higher resilience (Conbach's $\alpha=0.856$ ).

\section{Propensity score matching (PSM)}

The R version 3.6.3 software program (R Foundation for Statistical Computing, Vienna, Austria) was used to perform PSM. To adjust for the potential confounders between study and control groups, we applied PSM which used the nearest-neighbor matching algorithm with a 1:3 matching scheme for matching via R package "Matching". The propensity score was estimated_using the 13 potential confounders (e.g. age, gender, BMI, ethnicity, marital status, education level, employment status, monthly household income, tobacco use, alcohol use, frequency of hair washing, using Hair dryer/curling iron or hair straightener and hair styling products). The 1:3 PSM matched 20 participants in the control group and 60 PLWH in the study group. Lastly, we examined the standardized differences among the 13 confounders before and after PSM using the R package "Tableone".

\section{Statistical analysis}

Descriptive statistics were reported on the variables of interest. Categorical variables were described using frequencies and percentages, whereas the continuous variables were described using median and interquartile range (IQR). Differences in quantitative results were analyzed using the Pearson's Chi-squared or Fisher's exact tests, while those in semi-quantitative results were analyzed using the Mann-Whitney U test.

We examined the Spearman correlations between hair cortisol and psychological measures. To assess the differences of psychological measures between different levels of hair cortisol, the hair cortisol level was categorized into to two sub-groups ("low group": $\leq 29.56$, "high group": $>29.56$ ) using the median values of cortisol. Mann-Whitney $U$ test was employed to examine the associations between psychological measures and hair cortisol level.

All statistical analyses were conducted using the Statistical Pack for the Social Science (SPSS) version 23.0 software program (IBM Corp, Armonk, NY, USA). P values are two-tailed, and values less than 0.05 were considered statistically significant. 


\section{References}

1. Ortblad, K.F. et al. The burden of HIV: insights from the Global Burden of Disease Study 2010. AIDS 27, 2003-2017 (2013).

2. https://apps.who.int/gho/data/view.main.22100WHO?lang=en.

3. McIntosh, R.C. et al. Stress and coping in women living with HIV: a meta-analytic review. AIDS Behav 16, 2144-2159 (2012).

4. Napravnik, S., Royce, R., Walter, E. \& Lim, W. HIV-1 infected women and prenatal care utilization: barriers and facilitators. AIDS patient care and STDs 14, 411-420 (2000).

5. Hellhammer, D.H., Wust, S. \& Kudielka, B.M. Salivary cortisol as a biomarker in stress research. Psychoneuroendocrinology 34, 167-171 (2009).

6. Miller, G.E., Chen, E. \& Zhou, E.S. If it goes up, must it come down? Chronic stress and the hypothalamic-pituitary-adrenocortical axis in humans. Psychol Bull 133, $25-45$ (2007).

7. Liu, A.Y. et al. Strong relationship between oral dose and tenofovir hair levels in a randomized trial: hair as a potential adherence measure for pre-exposure prophylaxis (PrEP). PloS one 9, e83736 (2014).

8. Russell, E. et al. Toward standardization of hair cortisol measurement: results of the first international interlaboratory round robin. Ther Drug Monit 37, 71-75 (2015).

9. Chao, H., Cui Z.Y., Wang, Y., Li, L. \& Huang, H.H. Analysis of mental health status among people with HIV/AIDS. Chin J Hum Sex 26, 118-121 (2017).

10. Sinicco, A. et al. Cytokine network and acute primary HIV-1 infection. AIDS 7, 1167-1172 (1993).

11. Tao, W., Feng, X.S. \& Wu, Y.C. A Method to Estimate the Hazard Ratio in Non-randomized Medical Researehes. Chi J Heal Sta 21, 38-41 (2004).

12. Langerak T. et al. The relation between long-term cortisol levels and the metabolic syndrome in HIV-infected patients. Clin Endocrinol 83, 167-72 (2015).

13. Gong, S.L. Depression. Beijing: People's Medical Publishing House, 33 (2003).

14. Wan, Y.J., Dong, H.Y., Zhan, Y., Zhang, R.F. \& Lu, L.X. The mental problems and needs in patients under AIDS/HIV discrimination. Chin Rem \& Clin 7, 524-526 (2007).

15. Chen, Q.L. et al. Effect of Mental Health and Psychosocial Factors in Individual with HIV/AIDS. Chin Ment Heal J 12, 850-853 (2004).

16. Antoni, M.H. et al. Cognitive-behavioral stress management reduces distress and 24-hour urinary free cortisol output among symptomatic HIV-infected gay men. Ann Behav Med 22, 29-37 (2000).

17. Villette, J.M. et al. Circadian variations in plasma levels of hypophyseal, adrenocortical and testicular hormones in men infected with human immunodeficiency virus. J Clin Endocrinol Metab 70, 572-577 (1990).

18. Qiao S. et al. Hair Measurements of Cortisol, DHEA, and DHEA to Cortisol Ratio as Biomarkers of Chronic Stress among People Living with HIV in China: Known-Group Validation. PloS One 12, $\mathrm{e} 0169827$ (2017).

19. Odeniyi, I.A., Fasanmade, O.A., Ajala, M.O \& Ohwovoriole, A. E. Adrenocortical function in Nigerians with human immunodeficiency virus infection. Ghana Med J 47, 171-177 (2013).

20. Zaid, D \& Greenman, Y. Human Immunodeficiency Virus Infection and the Endocrine System. Endocrinol Meta 34, 95-105(2019).

21. Dettenborn L. et al. Introducing a novel method to assess cumulative steroid concentrations: increased hair cortisol concentrations over 6 months in medicated patients with depression. 
Stress 15, 348-353 (2012).

22. Karlen, J., Ludvigsson, J., Frostell, A., Theodorsson, E \& Faresjo, T. Cortisol in hair measured in young adults - a biomarker of major life stressors? BMC Clin Pathol 11, 12 (2011).

23. Steudte, S. et al. Increased cortisol concentrations in hair of severely traumatized Ugandan individuals with PTSD. Psychoneuroendocrinology 36, 1193-1200 (2011).

24. Staufenbiel, S.M., Penninx, B.W., Spijker, A.T., Elzinga, B.M., \& van Rossum, E.F. Hair cortisol, stress exposure, and mental health in humans: a systematic review. Psychoneuroendocrinology 38, 1220-1235 (2013).

25. Yekta, D. et al. Relationship between hair cortisol concentrations and depressive symptoms in patients with coronary artery disease. Neuropsychiatr Dis Treat 6, 393-400 (2010).

26. Manenschijn, L. et al. Long-term cortisol in bipolar disorder: associations with age of onset and psychiatric co-morbidity. Psychoneuroendocrinology 37, 1960-1968 (2012).

27. Steudte, S. et al. Decreased hair cortisol concentrations in generalised anxiety disorder. Psychiatry Res 186, 310-314 (2011).

28. Akoku, D.A., Tihnje, M.A., Tarh, E.O., Tarkang, E.E \& Mbu, R.E. Predictors of willingness to accept pre-marital HIV testing and intention to sero-sort marital partners; risks and consequences: Findings from a population-based study in Cameroon. PloS One, 13, e0208890 (2018).

29. Sauve, B., Koren, G., Walsh, G., Tokmakejian, S. \& Van, Uum, S.H. Measurement of cortisol in human hair as a biomarker of systemic exposure. Clin Invest Med 30, 183-191 (2007).

30. Qi, X. et al. Relationship between effort-reward imbalance and hair cortisol concentration in female kindergarten teachers. J Psychosom Res 76, 329-332 (2014).

31. Gao, W. et al. Quantitative analysis of steroid hormones in human hair using a column-switching LC-APCI-MS/MS assay. J Chromatogr B, Analyt Technol Biomed and life Sci 928, 1-8 (2013).

32. Sun, W., Wu, M., Qu, P., Lu, C \& Wang, L. Psychological well-being of people living with HIV/AIDS under the new epidemic characteristics in China and the risk factors: a population-based study. Int J Infect Dis 28, 147-152 (2014).

33. Cohen, S., Kamarck, T. \& Mermelstein, R. A global measure of perceived stress. J Heal Soc Behav 24, 385-396 (1983).

34. Lee, E.H. Review of the psychometric evidence of the perceived stress scale. Asian Nurs Res 6, 121-127 (2012).

\section{Acknowledgements}

This research was supported by grants from the National Natural Science Foundation of China (No. 81761128004 and R01MH0112376).

\section{Author contributions}

Riying Lv, Zhiyong Shen and Xiaoming Li designed the study. Xu Chen and Chengbo Zeng performed data analysis. Shuaifeng Liu and Shan Qiao were involved in the clinical aspects of the study. Xu Chen wrote the initial drafts of the manuscript and all authors contributed to editing and improving the manuscript.

\section{Corresponding authors}

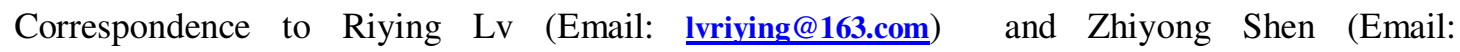
shenzhiyong99999@sina.com)

Competing interests 
The authors declare no competing interests. 
Figures

Raw Treated

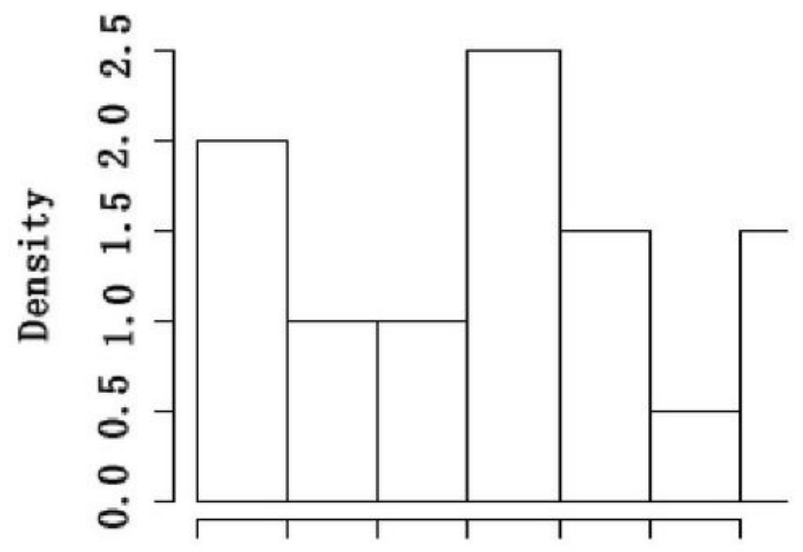

$\begin{array}{lllllll}0.0 & 0.1 & 0.2 & 0.3 & 0.4 & 0.5 & 0.6\end{array}$

Propensity Score

Raw Control

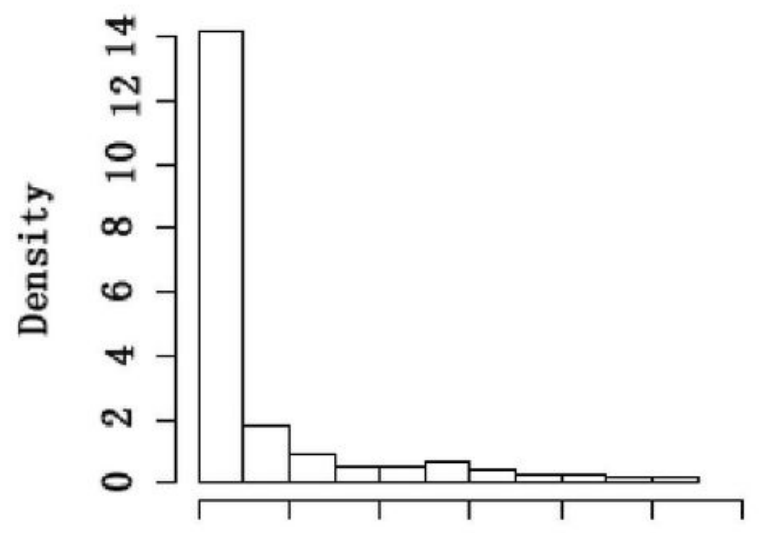

$\begin{array}{lllllll}0.0 & 0.1 & 0.2 & 0.3 & 0.4 & 0.5 & 0.6\end{array}$

Propensity Score
Matched Treated

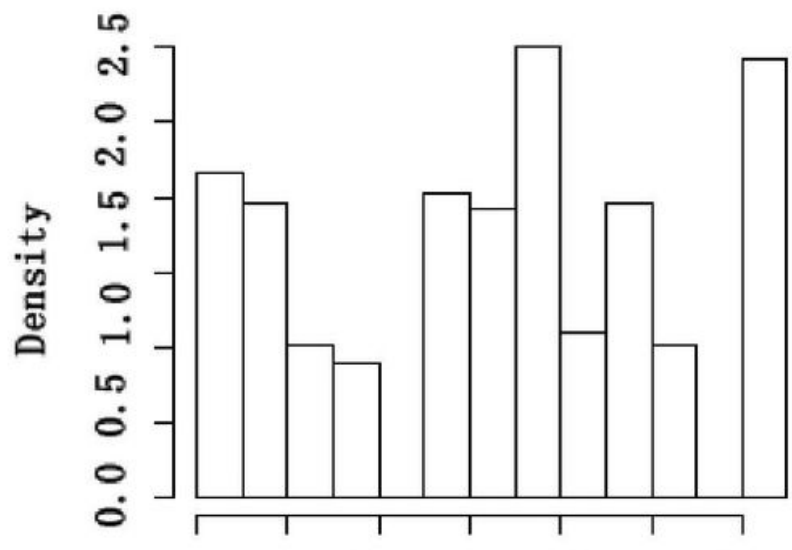
$\begin{array}{lllllll}0.0 & 0.1 & 0.2 & 0.3 & 0.4 & 0.5 & 0.6\end{array}$

Propensity Score

Matched Control

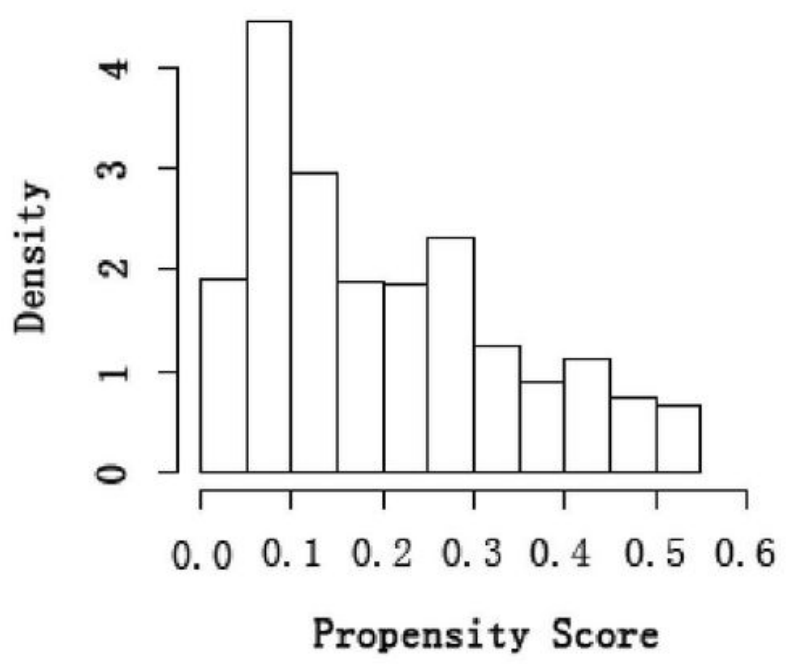

Figure 1

Histograms of the unmatched and matched participants. Note: The overall balance of the thirteen variables in the model indicates that the matching process was successful. 
a

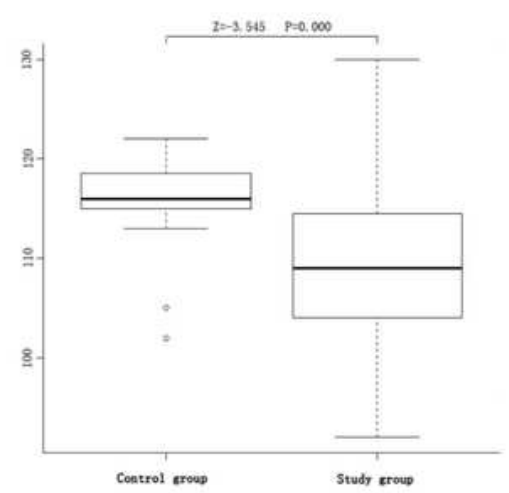

c

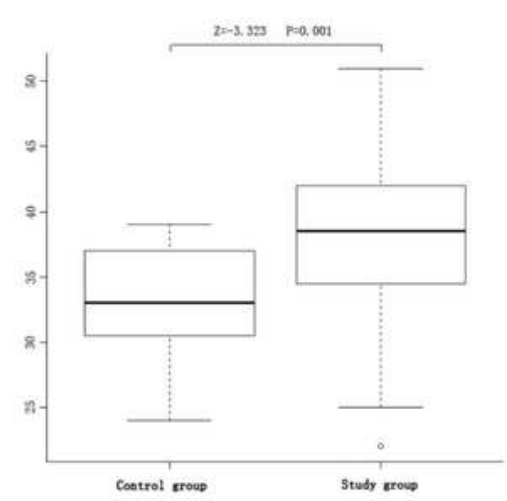

e

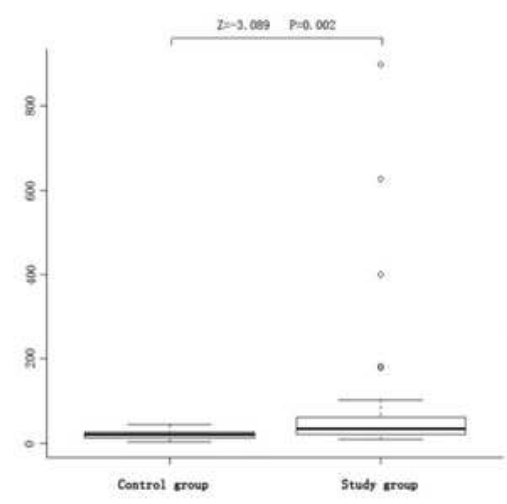

b

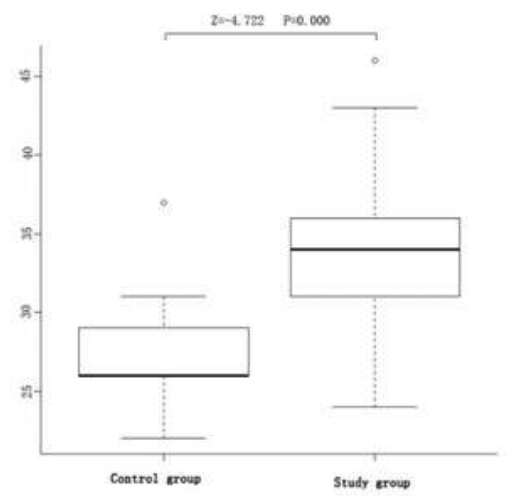

d

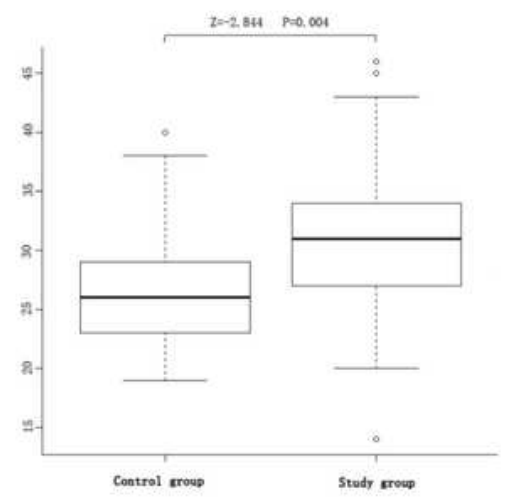

\section{Figure 2}

Comparison of psychological stress level and cortisol level between control and study groups after propensity matching. (a-d) The level of quality life, anxiety, perceived stress, and psychological resilience among PLWH and without HIV, respectively. (e) The level of cortisol among PLWH and without HIV. 